\title{
El tratamiento de las demencias en el sistema de salud de Uruguay
}

\author{
Dementias: Treatment within Uruguayan Health \\ System
}

\section{Tratamento das demências no sistema de saúde do Uruguai}

\author{
Robert Pérez Fernández \\ ORCID ID: 0000-0001-7388-8600 \\ Instituto de Psicología Social, Facultad de Psicología \\ Centro Interdisciplinario de Envejecimiento \\ Universidad de la República, Uruguay
}

Autor referente: rperez@psico.edu.uy

Historia Editorial

Recibido: 16/03/2018

Aceptado: 21/06/2018

\section{RESUMEN}

La Organización Mundial de la Salud ha exhortado a los gobiernos del mundo a declarar la demencia como una prioridad de salud pública. Recientemente ha definido un plan de acción mundial sobre la respuesta de salud pública a la demencia 2017-2025. El objetivo del presente estudio es conocer la respuesta que da el sistema de salud de Uruguay en materia de tratamientos de la demencia, aportando al área de tratamientos y atención de dicho plan. Se diseña un estudio descriptivo e interpretativo basado en las trayectorias de atención, que utiliza métodos cuantitativos y cualitativos para estudiar dos poblaciones: familiares y médicos. Los principales resultados indican que el sistema de salud de Uruguay es accesible para las personas con demencia, pero da una respuesta muy limitada en cuanto a tratamientos, no cumpliendo con el estándar de calidad y buenas prácticas definido internacionalmente.

tratamiento brindado es exclusivamente farmacológico y no existe disponibilidad de tratamientos psicosociales dentro del propio sistema de salud. De esta forma, todos los aspectos de la enfermedad-cognitivos, afectivos, conductuales, de sufrimiento psíquico-son abordados de forma exclusivamente neuroquímica, lo que hace muy ineficiente al propio sistema. Las personas con demencia atendidas presentan un alto consumo de fármacos psicotrópicos, incluido 
algunos que están especialmente contraindicados por sus efectos secundarios. Se discuten estas estrategias de atención como parte de un dispositivo biopolítico y una perspectiva de enfermedad objetivo- natural, que implican un riesgo de iatrogenia. Finalmente, se realizan algunas recomendaciones para revertir esta situación y mejorar el sistema de salud.

\section{Palabras clave: Demencia; Enfermedad de Alzheimer; Psicofármacos; Tratamiento}

\section{ABSTRACT}

World Health Organization has called Governments to make dementia a public health priority. A global action plan on the public health response to dementia 2017-2025 has recently been defined. The aim of the present study is to know the response given by the Uruguayan health system regarding treatments for dementia, addressing to areas of this plan. A descriptive and interpretative study is designed based on care trajectories; it uses quantitative and qualitative methods to study two populations: family and doctors. The main results indicate that the health system of Uruguay is accessible to people with dementia, but it gives a very limited response in terms of treatments, not complying with the internationally defined standards of quality and good practice. The treatment provided is exclusively pharmacological and there is no availability for psychosocial treatments within the health system itself. In this way, all aspects of the diseasecognitive, affective, behavioral, psychic suffering, etc.-are dealt within an exclusively neurochemical way, which makes the system itself very inefficient. People treated for dementia show a high consumption of psychotropic drugs, including some that are especially contraindicated due to their side effects. These care strategies are discussed as part of a biopolitical device and a perspective of objectivenatural disease, which imply a high risk of iatrogenesis. We conclude by making some recommendations to reverse this situation.

Keywords: Dementia; Alzheimer's disease; Psychotropic drugs; Treatment

\section{RESUMO}

A Organização Mundial da Saúde convidou os governos do mundo a declararem a demência como uma prioridade de saúde pública. Recentemente, definiu um plano de ação global sobre a resposta da saúde pública à demência 2017-2025. O objetivo deste estudo é conhecer a resposta do sistema de saúde uruguaio em termos de tratamentos $\mathrm{da}$ demência, contribuindo para a área de terapêuticas e cuidados desse plano. Este estudo, descritivo e interpretativo com base em trajetórias das assistência que utilizam métodos quantitativos e qualitativos, estudou duas populações: família e médicos. Os principais resultados indicam que o sistema de saúde do Uruguai é acessível para pessoas com demência, mas dá uma resposta muito limitada em termos de tratamentos, não cumprindo a qualidade internacional e o padrão das boas práticas. O que é fornecido é exclusivamente farmacológico e não há disponibilidade de tratamentos psicossociais dentro do próprio sistema de saúde. Desta forma, todos os aspetos da doençasofrimento cognitivo, afetivo, 
comportamental, psíquico, etc. 一são tratados de forma exclusivamente neuroquímica, tornando muito ineficiente 0 próprio sistema. As pessoas com demência tratadas apresentam alto consumo de drogas psicotrópicas, incluindo algumas que são especialmente contraindicadas devido aos efeitos colaterais. Essas estratégias de atenção são discutidas como parte de um dispositivo biopolítico e uma perspetiva de doença meta-natural, implicando um alto risco de iatrogenia; e fazendo algumas recomendações para reverter essa situação.

Palavras chave: Demências; Doença de Alzheimer; Psicofármacos; Tratamento

\section{Introducción}

as demencias constituyen un síndrome producido por diferentes enfermedades que afectan el cerebro, en general de forma crónica o progresiva, generando déficits en distintas funciones de alta integración cortical, como la memoria, el lenguaje, las funciones ejecutivas, etc.. Es común que estos déficits se acompañen o sean precedidos por una afección en el control emocional o en el comportamiento social. La enfermedad de Alzheimer (EA) es la causa más común de demencia ya que sus rasgos histopatológicos se encuentran presentes en la mayoría de los casos, ya sea en forma exclusiva o con otras alteraciones (Organización Mundial de la Salud [OMS], 2013).

Genéricamente, las demencias se consideran dentro de los principales trastornos crónicos que producen discapacidad y dependencia entre las personas mayores, siendo responsables de casi el $12 \%$ de los años vividos con discapacidad a causa de una enfermedad no transmisible (Alzheimer's Disease International [ADI], 2015). Si bien no son trastornos exclusivos de la vejez, a partir de los 60 años de edad duplican cada cinco años su prevalencia, afectando al 8,5\% de las personas mayores de esa edad (ADI, 2013). En un mundo cuya población es cada vez más envejecida, en el año 2015 se estimaba que había casi 47 millones de personas con demencia, cifra que se duplicaría cada 20 años. Sin embargo ese incremento no será homogéneo, sino que será mayor en los países de renta baja y media—como la mayoría de América 
Latina-acorde con el mayor envejecimiento poblacional que tendrán en los próximos años (ADI, 2015).

Teniendo en cuenta esa situación, la OMS ha realizado un llamado a los gobiernos del mundo para que declaren las demencias como una prioridad de salud pública (OMS, 2013). Para implementar esa resolución, se ha diseñando el plan de acción mundial sobre la respuesta de salud pública a la demencia, para el período 2017-2025, que incluye un área de actuación en diagnóstico, tratamiento y atención (OMS, 2017).

En relación al tratamiento de las demencias y los deterioros cognitivos, la literatura es congruente en señalar los beneficios de realizar un abordaje mixto: farmacológico y no farmacológico o psicosocial (Allegri et. al., 2010; Alzheimer's Society, 2013 y 2014; Department of Health, 2009; Ministerio de Sanidad, Política Social e Igualdad de España [MSPSI], 2010; National Institute for Health and Clinical Excellence [NICE], 2007; OMS, 2013). El tratamiento farmacológico de la demencia-o tratamiento antidemencial-actualmente consiste en el uso de fármacos anticolinesterásicos en la fase leve-moderada y memantina-antagonista de los receptores NMDA—en fase moderada/avanzada. A su vez, para los llamados síntomas conductuales y psicológicos asociados a la demencia, las guías clínicas señalan como primer medida el ubicar y tratar posibles causas subyacentes de los mismos, tales como infecciones $u$ otras enfermedades médicas. Como segunda línea de intervención indican utilizar medidas de entorno y tratamientos psicosociales $y$, si con estas dos acciones no se consiguen mejoras, iniciar un tratamiento farmacológico controlado con psicofármacos específicos, tales como antipsicóticos, antidepresivos, etc., comenzando con dosis menores y por un tiempo prudencial. Por su parte, el tratamiento no farmacológico o psicosocial, es definido como "cualquier intervención no química, focalizada y replicable, sustentada sobre una base teórica, realizada en el paciente o el cuidador, con capacidad potencial de lograr algún beneficio relevante" (Olazarán et. al., 2010, p. 
2). Corresponden a esta categoría diferentes intervenciones que incluyen desde ejercicio físico, psicoeducación, terapias de tipo ocupacional o de estimulación cognitiva, a tratamientos psicoterapéuticos como terapias de reminiscencia, cognitivasconductuales o psicodinámicas (Cabrera et. al., 2015; Cammisuli, Danti, Bosinelli \& Cipriani, 2016; Olazarán et. al., 2010).

El enfoque de tratamiento farmacológico predomina ampliamente en la literatura y se encuentra muy bien protocolizado. No obstante eso, hasta el momento han sido muy limitados sus beneficios, más teniendo en cuenta que presentan la posibilidad de generar efectos secundarios (Allegri et. al., 2010; Alzheimer's Society, 2013; Cabrera et. al., 2015; MSPSI, 2010; NICE, 2007; Olazarán et. al., 2010; OMS, 2013). Se han reportado algunos beneficios de este tipo de tratamiento en enlentecer por un tiempo el declive cognitivo en pacientes en fase inicial o media (Homma et. al., 2008; Burns et. al., 1999). No se han reportado beneficios en la mejora de la calidad de vida de las personas con demencia (Cooper et. al., 2013) ni se ha encontrado evidencia concluyente de su efectividad en el tratamiento de los síntomas neuropsiquiátricos (Human Rights Watch, 2018; Sink, Holden \& Yaffe, 2005).

Respecto a los tratamientos psicosociales, no existe una evidencia concluyente sobre su efectividad. Asimismo, tampoco existe consenso respecto a la calidad de los datos, ya que la falta de apoyo financiero para investigar estas propuestas, hacen que las mismas en general presenten una serie de limitaciones metodológicas para su estudio (Cammisuli et. al., 2016; Brasure et. al., 2016; Olazarán et. al., 2010). No obstante este panorama global, algunas intervenciones específicas han demostrado ser efectivas en la mejora de la calidad de vida (Cabrera et. al., 2015; Cheston \& Ivanecka, 2017; Cooper et. al., 2012; Olazarán et. al., 2010), en reducir los síntomas conductuales y psicológicos (Brodaty \& Arasaratnam, 2012; Livingston et. al., 2014; Olazarán et. al., 2010; Richter, Meyer, Möhler \& Köpke, 2012), en disminuir síntomas conductuales y 
depresión (García-Alberca, 2015; O’Neil et. al., 2011), en la mejora cognitiva, del estado de ánimo y en las Actividades de la Vida Diaria (Olazarán et. al., 2010). En ninguno de estos casos se han reportado efectos secundarios y algunos señalan efectos al menos equivalentes a los de la farmacoterapia (Brodaty \& Arasaratnam, 2012; Olazarán et. al., 2010). Toda la literatura consultada señala la necesidad de profundizar la investigación en esta línea.

A partir de este estándar de calidad definido para los tratamientos de las demencias, el presente trabajo tiene como principal objetivo conocer cuál es la respuesta que actualmente está dando el Sistema Nacional Integrado de Salud de Uruguay (SNIS) en materia de tratamientos para las personas con demencia.

Uruguay, con un 14\% de su población con 65 o más años (Instituto Nacional de Estadística, 2018), es el país más envejecido de América del Sur. Estudios internacionales estiman en 54.000 las personas con demencia en el país (ADI, 2013), lo que significa casi un $11 \%$ de las personas de 65 años o más. Respecto a los costes, en el año 2010 se destinó algo más del uno por ciento del Producto Bruto Interno a gastos de asistencia médica, apoyo social y cuidado informal de las personas con demencia (ADI, 2013). Sin embargo, a pesar de esas cifras, el tema de las demencias y el deterioro cognitivo hasta el momento ha ocupado un lugar muy marginal dentro de las políticas públicas y de salud de Uruguay, no existiendo aún acciones específicas en ningunas de las áreas definidas en el plan de acción mundial sobre la respuesta de salud pública a la demencia. De ahí la importancia de comenzar a producir conocimientos de la situación del país en relación a las metas definidas por la OMS.

\section{Metodología}

Este trabajo forma parte de un estudio más amplio sobre las prácticas de atención y cuidado para personas con demencia que se implementan en Uruguay (Pérez, 2016). 
Se trata de un estudio descriptivo e interpretativo, en base a un diseño que realiza una triangulación de métodos y poblaciones. Para la producción y procesamiento de los datos se utilizaron métodos cuantitativos y cualitativos, teniendo como fuente de información una población de familiares de personas con demencia y otra de médicos a cargo de la atención.

El diseño global del proyecto se basa en el estudio de las trayectorias de atención (patway to care), enfoque de larga tradición en salud mental, que busca reconstruir de manera organizada los pasos que va dando una persona con una determinada enfermedad, desde las primeras manifestaciones hasta el diagnóstico (Gater, et. al., 1991). En el campo de las demencias ha sido utilizado con algunas adaptaciones en investigaciones realizadas principalmente en EEUU (p.e. Boise, Morgan, Kaye, \& Camicioli, 1999), Australia (Speechly, Bridges-Webb \& Passmore, 2008), Reino Unido (p.e. Chrisp, Thomas, Goddard, \& Owens, 2011), Italia (Cattel et. al., 2000) y China (p.e. Zhao et. al., 2016). No he encontrado reportes de estudios realizados anteriormente en América Latina.

\section{Procedimientos y técnicas}

En una primera fase se contactó y entrevistó a familiares cuidadoras informales de personas que estuvieran siendo tratadas por algún tipo de demencia en el sistema de salud. Como instrumento de recolección de la información se utilizó la entrevista retrospectiva (Drebing et. al., 2004). Las entrevistas se realizaron de forma presencial por medio de un entrevistador especialmente entrenado. La pauta diseñada contiene 54 preguntas de información cuantitativa (fechas, tiempos de demora, tratamientos, cantidad y tipo de fármacos administrados en los diferentes momentos, etc) y cualitativas (vinculadas a eventos y significados sobre lo que estaba sucediendo). En el presente trabajo se comunican únicamente los resultados sobre tratamientos. 
Luego de obtenidos los primeros resultados de las entrevistas a las familiares, los mismos fueron presentados y discutidos grupalmente con ellas a los efectos de revisar los datos. Las ideas y propuestas emergentes de estos encuentros fueron incorporadas al análisis. Los datos cuantitativos se procesaron por medio del software SPSS versión 20, utilizando estadística descriptiva para un primer nivel de análisis, y estadística inferencial en un segundo nivel, a los efectos de evaluar la asociación entre variables relevantes por medio de las pruebas chi cuadrado $(\mathrm{X} 2)$ y comparar diferentes grupos utilizando análisis de varianza (ANOVA). La probabilidad $=<0.05$ fue considerada significativa. Se identificaron cuatro casos con valores atípicos (outliers) que fueron eliminados.

Una vez finalizada esa fase, se procedió a seleccionar y entrevistar a profesionales médicos que estuvieran desempeñándose dentro del sistema de salud en la atención de personas con demencia. En este caso se utilizó como instrumento la entrevista cualitativa de tipo semidirigida, efectuada en forma presencial. Esta información se procesó por medio del software Atlas.ti, versión 6.0.15, realizándose un análisis de contenido temático cualitativo.

\section{Participantes}

Para referirme a las/os familiares_cuidadores/as principales, utilizaré el femenino como genérico-tanto para hombres como para mujeres-pues como se verá, en nuestra muestra existe un amplio predominio de mujeres. A su vez, para referirme a los profesionales entrevistados, utilizaré el masculino como genérico-tanto para hombres como para mujeres-por ser mayoría los hombres y como forma de preservar la identidad de cada profesional.

Se realizó un muestreo de tipo intencional. La muestra quedó conformada por 116 familiares cuidadoras informales (FC) y 15 profesionales médicos (tabla 1). Las FC fueron reclutadas a partir de diversas fuentes: agrupaciones de familiares de diferentes 
ciudades, profesionales, grupos de personas mayores, servicios universitarios, servicios médicos, etc. El criterio de inclusión fue ser FC (no rentada) de una persona que estuviera siendo tratada por algún tipo de demencia o EA en el sistema de salud. Se utilizaron ambos términos como forma de hacerlo más accesible a las familiares, teniendo en cuenta la heterogeneidad de términos que ellas manejan sobre el diagnóstico (demencia, EA, demencia senil, etc.). En todos los casos se chequeó que efectivamente su familiar cumplía con los criterios de demencia de la OMS (2013). Para conformar esta muestra se buscó que el grupo de personas con demencia estudiadas mantuvieran un cierto equilibrio interno en relación a la población en general, en cuanto a nivel educativo, ingresos y tipo de cobertura de atención en salud. Asimismo, se buscó activamente incluir casos de personas que residieran en departamentos con alta, media y baja densidad de población mayor. De esta forma, se entrevistó a FC en 18 ciudades, pertenecientes a ocho departamentos del país. 
Tabla 1

Características Sociodemográficas de las Personas con Demencia y sus FC

Personas con $\quad$ FC
demencia

\begin{tabular}{|c|c|c|c|}
\hline \multirow{2}{*}{ Sexo } & Hombres & 20 & 100 \\
\hline & Mujeres & 90 & 16 \\
\hline \multirow{3}{*}{ Escolaridad } & Primaria & 66 & 10 \\
\hline & Secundaria & 19 & 50 \\
\hline & Terciaria & 31 & 52 \\
\hline \multirow{3}{*}{ Ingresos } & Bajo & 25 & 43 \\
\hline & Medio & 48 & 64 \\
\hline & Alto & 41 & 7 \\
\hline \multirow{3}{*}{ Vive con... } & Familiar & 77 & \\
\hline & Residencial & 24 & \\
\hline & Solo & 15 & \\
\hline \multirow{2}{*}{ Prestador de salud } & Privado & 83 & \\
\hline & Público & 33 & \\
\hline
\end{tabular}

Las entrevistas se realizaron en diferentes lugares: domicilio, locales de la Asociación Uruguaya de Alzheimer y Similares (AUDAS), de la Universidad de la República, centros comunitarios, organizaciones de personas mayores, entre otros. Los encuentros grupales de presentación y discusión de los resultados dirigidos a las FC entrevistadas se realizaron entre los meses de agosto y setiembre del 2014 en las ciudades de Maldonado, Minas, Montevideo, Rosario, Salto y Tacuarembó.

Por su parte, la muestra de profesionales (tabla 2) quedó compuesta por 15 médicos que presentan un rango de edad de 33 a 76 años ( $m e d i a=49$, mediana=49, moda=34). 
Salvo un caso, los demás trabajaban en más de un prestador de salud o institución de asistencia, y se desempeñaban simultáneamente en el sistema público y privado. Las entrevistas se llevaron adelante en los lugares que los profesionales indicaban, que en general eran sus lugares de trabajo (hospital o policlínica), en horario previo o posterior a su consulta. Algunas entrevistas se realizaron en consultorios particulares, en locales comunitarios y de la Universidad de la República.

Tabla 2

Características de la muestra de Médicos

\begin{tabular}{|c|c|c|}
\hline & Medicina familiar & 4 \\
\hline & Psiquiatría & 4 \\
\hline \multirow[t]{5}{*}{ Especialización } & Neurología & 3 \\
\hline & Geriatría & 3 \\
\hline & Medicina general & 1 \\
\hline & Colonia & 2 \\
\hline & Maldonado & 2 \\
\hline Departamento en el que & Lavalleja & 2 \\
\hline \multirow[t]{6}{*}{ realizan asistencia } & Montevideo & 6 \\
\hline & Salto & 2 \\
\hline & Tacuarembó & 1 \\
\hline & Mujer & 7 \\
\hline & Hombre & 8 \\
\hline & Década de 1970 & 1 \\
\hline Década de egreso como & Década de 1980 & 5 \\
\hline \multirow[t]{2}{*}{ médico (título de grado) } & Década de 1990 & 4 \\
\hline & Primera década del S. XXI & 5 \\
\hline
\end{tabular}




\section{Aspectos Éticos}

El presente estudio fue avalado por el Comité de Ética en Investigación de la Facultad de Psicología de la Universidad de la República, Uruguay.

\section{Resultados}

No se pudo constatar ninguna asociación significativa entre los tratamientos implementados y las diferentes variables sociodemográficas analizadas, tales como edad, sexo, lugar de residencia, sistema público o privado de atención, nivel educativo o económico, etc. Esto indica que el sistema de salud de Uruguay es accesible y equitativo para las personas con demencia y trastornos cognitivos. Sin embargo, su respuesta es muy limitada en cuanto a tratamientos, pues al momento del diagnóstico, el $90 \%$ de los tratamientos indicados son exclusivamente farmacológicos o acompañados de sugerencias de actividades menores. Solo a un $5 \%$ de la muestra se le indica un tratamiento farmacológico y psicosocial (tabla 3).

Tabla 3

Tratamientos Indicados en el SNIS al Momento del Diagnóstico

\begin{tabular}{cccc}
\hline & & $(\mathrm{n})$ & $\%$ \\
\hline & Farmacológico & 88 & 79,3 \\
Tipo de & sugerencia de actividades & & \\
tratamiento & (crucigramas, ejercicios, & 12 & 10,8 \\
& etc.) & & \\
& Farmacológico y & 6 & 5,4 \\
Total & psicosocial & 5 & 4,5 \\
& Ningún tratamiento & 111 & 100 \\
\hline
\end{tabular}


Las guías clínicas consultadas coinciden en lo modesto de los resultados de los tratamientos farmacológicos aislados, señalando incluso el riesgo de algunos fármacos utilizados para síntomas asociados a la demencia. Esto es algo que no desconocen los médicos entrevistados:

...a esta altura uno ya viene de nuevo y sabe que no son tan efectivos, el tratamiento farmacológico especialmente. Los otros no, pero el tratamiento farmacológico [...] por lo menos hay que pensarlos un poco más, porque tienen efectos colaterales.... (Médico14)

Sin embargo, tal como está organizado el SNIS, no existe una opción terapéutica no farmacológica dentro del propio sistema. En los pocos casos que se indicaron tratamientos psicosociales, los mismos no fueron cubiertos dentro de las prestaciones del SNIS, por lo que cada familia debió gestionarlos por fuera del sistema. A su vez, parecería que la disponibilidad de tratamientos farmacológicos como única opción del sistema de salud de Uruguay forma parte de una práctica vinculada a las creencias y representaciones de la salud y enfermedad que emanan del modelo biomédico y su relación con la industria farmacéutica,

claro que está también, digo yo, la presión social y la presión de la industria.... pero yo veo que hoy en día se da donepecilo... yo que sé, como leche hervida. A la primera queja de memoria ya el médico le da un donepecilo sin saber o haberlo evaluado bien. (Médico 6)

En el contexto de un sistema altamente medicalizado cada persona con demencia consume un alto número de fármacos psicotrópicos como forma de gestión de la enfermedad. Como se visualiza en la tabla 4, dos tercios de la muestra consume tres o más medicamentos psicotrópicos. 
Tabla 4

Cantidad de Fármacos Psicotrópicos que Consumen las Personas con Demencia al Momento de la Entrevista

\begin{tabular}{|c|c|c|}
\hline № de fármacos & № de personas & $\%$ \\
\hline 1 & 7 & 6,0 \\
\hline 2 & 27 & 23,3 \\
\hline 3 & 25 & 21,6 \\
\hline 4 & 22 & 19,0 \\
\hline 5 & 9 & 7,8 \\
\hline 6 & 5 & 4,3 \\
\hline 7 & 3 & 2,6 \\
\hline 10 & 1 & 0,9 \\
\hline Casos perdidos & 17 & 14,7 \\
\hline Total & 116 & 100 \\
\hline
\end{tabular}

A este número de fármacos psicotrópicos se deben agregar otros no relevados en este estudio, que se consumen para otras patologías como gastritis, hipertensión, etc..

En este contexto de gestión farmacológica de la enfermedad, interesa especialmente conocer qué aspectos de la misma se gestionan de esta forma: ¿los cognitivos? ¿los afectivos? ¿la vida de relación?. Los resultados que se presentan en la tabla 5, indican que ya desde el diagnóstico se comienza una gestión farmacológica de los casos en los aspectos cognitivos (anticolinesterásicos y memantina), de la conducta (antipsicóticos) y del estado afectivo (antidepresivos y ansiolíticos). Estas tres áreas, vinculadas entre sí - pero a la vez diferenciables a los efectos de la intervención - para las FC forman parte del mismo cuadro orgánico de la demencia, naturalizando que el mismo debe gestionarse de forma exclusivamente bioquímica. Se reafirma así el 
modelo biomédico de comprensión de estos trastornos como un problema exclusivamente cerebral e individual.

Tabla 5

Fármacos Indicados como Tratamiento en el Momento del Diagnóstico y en el Momento de la Entrevista, en porcentaje (\%)

Diagnóstico Entrevista

\begin{tabular}{llcc}
\hline & Anticolinesterasicos & 46 & 52 \\
& Memantina & 39 & 42 \\
& Antipsicóticos & 46 & 57 \\
Tipo de fármaco & Benzodiacepinas & 33 & 44 \\
& Antidepresivos & 26 & 31 \\
& Neuroprotectores & 12 & 13 \\
& Otros & 31 & 37 \\
\hline
\end{tabular}

Cuando consultamos sobre esta situación de medicalización de las conductas a los médicos, las repuestas reafirman la perspectiva biomédica de la enfermedad, por lo que estos fármacos aparecen como la mejor forma de tratamiento:

en cuanto a tratamiento de la enfermedad de base, en este caso la EA, la utilización de inhibidores de la colinesterasa, memantina y todo el tratamiento de los síntomas accesorios o subsidiarios. Bueno, si el paciente está deprimido se le indica antidepresivos. Si esta agitado, que es algo muy frecuente, sobre todo en las etapas avanzadas, utilizamos antipsicóticos. Y también la no utilización de - porque eso también es tratar de evitar la iatrogenia - de tratar de evitar el uso benzodiacepinas. (Médico 13)

Parecería que ante la ausencia de otro tipo de tratamiento, los fármacos pasan a utilizarse como sujeción química para controlar las conductas de las personas. Cuando 
comenté a los médicos entrevistados los resultados de algunos estudios que plantean contraindicaciones para emplear antipsicóticos, antidepresivos y ansiolíticos, casi todos contra-argumentaron eso:

Justo venimos recién de esa charla que dio el doctor x (reconocido profesional del ambiente médico de Uruguay) y que sí, en ningún momento en el paciente con demencia se plantea el uso de benzodiacepinas. Siempre antipsicóticos y neurolépticos, incluso en dosis bajas se plantea el uso de neurolépticos. [Entrevistador: ¿neurolepticos clásicos?] Clásicos si. El profesor recién planteaba que uno de los protocolos para el manejo en agudos es la combinación de risperidona con dosis bajas de haloperidol [...] [Entrevistador: en el tema de los antipsicóticos hay algunas revisiones que los desaconsejan por su efecto a nivel vascular [...] Y es como todo, que también viste, el costo-beneficio. Eso lo medimos mucho en medicina, porque [...] si me rompe toda la casa, poco me va importar si después aumentará la incidencia de arritmia. (Médico 13)

A su vez, las FC de las personas con demencia forman parte y sostienen este sistema de creencias y concepciones biomédicas de la enfermedad. Cuando les preguntamos qué es la demencia, la mayoría respondió con conceptos similares a éstos:

La definiría como una enfermedad en cual existe un deterioro, prácticamente en toda la actividad cerebral, un deterioro total Familiar 25

Y no sé... como una... digo, palabras poco profesionales por supuesto, porque yo no vengo desde lo científico, como una muerte...yo que sé...neuronal... (Familiar 55)

En este sistema de creencias y concepciones de la demencia, es común que los propios pacientes le exijan al médico la utilización de fármacos como primer línea de tratamiento y tiendan a menospreciar otro tipo de propuesta. 
Y sí, porque lo que los familiares quieren y lo que los cuidadores quieren es que el paciente este tranquilo. Entonces como médicos, en realidad [...] los viejitos que yo veo, la mayoría de la medicación que reciben, la quetiapina es una de las que más se usan. (Médico 9)

\section{Discusión}

El tratamiento para las demencias que brinda el sistema de salud de Uruguay es exclusivamente farmacológico, no habiendo disponibilidad de tratamientos psicosociales dentro del propio sistema. En ese sentido, el país actualmente no cumple con el estándar de calidad señalado en las guías de buenas prácticas. Como ha planteado João Biehl, la disponibilidad de fármacos es importante para lograr un sistema de salud eficiente, "mas é também o vetor de uma crescente farmacolização da saúde pública em detrimento de outras iniciativas preventivas e de infra-estrutura" (2008, p. 440) . Algo de esto es lo que sucede en Uruguay: si bien el SNIS brinda un tratamiento farmacológico accesible, al ser la única respuesta de abordaje termina siendo muy limitada y altamente ineficiente en el campo de la salud mental.

La alta medicalización del sistema se puede apreciar claramente si se compara la cantidad y el tipo de fármacos utilizados para la gestión de las demencias en Uruguay, con lo que sucede en otros países que han desarrollado políticas públicas de abordaje y buenas prácticas. Por ejemplo, en el reciente estudio de Birmingham (Inglaterra), en una muestra de 1000 personas con demencia realizada en una fecha similar al estudio de Uruguay, obtienen que el $20 \%$ de los pacientes reciben antipsicóticos, $11 \%$ antidepresivos, $11 \%$ benzodiacepinas, $21 \%$ inhibidores de la acetilcolinesterasa y $22 \%$ memantina (Bangash et. al, 2017). Sin embargo, en la muestra de Uruguay tienen indicado cuatro veces más benzodiacepinas, casi tres veces más antipsicóticos y antidepresivos, y dos veces más fármacos antidemenciales (memantina y 
anticolinesterásicos). He aquí el ejemplo de dos respuestas de tratamiento diferentes ante los mismos trastornos.

La baja eficiencia del tratamiento exclusivamente farmacológico es más evidente a medida que va transcurriendo el tiempo y la enfermedad va avanzando, ya que los únicos movimientos terapéuticos posibles dentro de este universo limitado son la modificación de la dosis o la incorporación de nuevas drogas. El tipo de fármacos que se va agregando es principalmente para la gestión de las conductas y estado de ánimo. Así, ante los conflictos que se empiezan a generar en la vida cotidiana de las personas con demencia - muchas veces por falta de apoyo y que rápidamente son decodificados por el sistema de salud como trastornos de conducta por la enfermedad - inmediatamente se aumenta el consumo de antipsicóticos. A su vez, las repercusiones anímicas que esta situación va produciendo, son abordadas introduciendo - o aumentando la dosis de - antidepresivos y benzodiacepinas.

Debe tenerse presente que esta gestión farmacológica interactúa químicamente entre sí, muchas veces con efectos iatrogénicos. En el caso de los fármacos antipsicóticos, si bien en diferentes lugares del mundo se utilizan como parte del tratamiento, existen una serie de estudios que señalan la prudencia que se debe tener por sus efectos adversos, tales como el exceso de sedación, temblores, riesgo de caídas, infecciones respiratorias, aumento del deterioro cognitivo y riesgo elevado de factores cerebrovasculares (Alzheimer's Society, 2011; Ballard, Waite \& Birks, 2012; Human Rights Watch, 2018). De ahí que se desaconseje enfáticamente su uso como primera opción ante los trastornos de conducta, ubicando su empleo de forma selectiva en una tercera línea. Si se aplicara este criterio de buenas prácticas, en Uruguay sólo se les indicaría este tipo de fármacos a un pequeño grupo de las personas con demencia. Eso implicaría gestionar de una forma más adecuada los conflictos vinculares que se 
presentan habitualmente en estas enfermedades, introduciendo diferentes modalidades de tratamientos psicosociales.

Lo mismo sucede con las benzodiacepinas, que tampoco son aconsejadas como primera opción de tratamiento por sus efectos secundarios. Sobre este tipo de fármaco, en los médicos entrevistados parece haber consenso sobre sus efectos negativos. Sin embargo, no deja de sorprender la alta cantidad de personas con demencia de la muestra que tienen prescripto este tipo de medicamento, que pasa a ser el tercer fármaco más consumido (casi la mitad de la muestra). Parece haber aquí un desfasaje entre los conocimientos sobre los efectos secundarios y la prescripción que se realiza en la práctica clínica cotidiana.

Respecto a los fármacos antidepresivos, si bien en nuestro medio parece ser indiscutido su beneficio terapéutico, existen estudios que cuestionan su eficacia bioquímica, planteando que sus beneficios terapéuticos están más relacionados al "efecto placebo" que a los efectos de la droga en sí (Kirsch et. al., 2008). Cuando se han estudiado en personas con demencia y depresión el efecto de dos tipos de antidepresivos - sertralina como inhibidor selectivo de la recaptación de serotonina y mirtazapina, fármaco noradrenérgico y serotoninérgico - tampoco se han encontrado diferencias significativas entre estos fármacos y el placebo. Por el contrario, el grupo placebo de este estudio presentó una reducción significativa de los síntomas de depresión similar a la de los grupos con antidepresivos, con la ventaja adicional de que no sufrieron ningún efecto secundario (Banerjee et. al., 2013).

A pesar de estos estudios y conocimientos disponibles, en el sistema de salud de Uruguay se insiste con el tratamiento farmacológico como única opción. Las concepciones biomédicas de la enfermedad por parte de médicos y familiares, junto a la falta de tratamientos no farmacológicos para abordar los trastornos que se producen en la convivencia con las personas con demencia, van naturalizando un tipo de 
intervención que termina produciendo un efecto de sujeción química (Sociedad Española de Geriatría y Gerontología, [SEGG], 2014) o de chemical restraints, en términos de Human Rights Watch (2018). Las mismas refieren al uso de fármacos para controlar sin el consentimiento del paciente, aspectos de la conducta en general que podrían abordarse de otra forma que no violentara sus derechos humanos. Se utilizan aquí fármacos que actúan sobre el sistema nervioso central, limitando en los pacientes su funcionamiento mental en general, la movilidad y las actividades de la vida diaria (SEGG, 2014). Este tipo de restricciones es habitual que se utilice en personas con demencia que viven en instituciones de larga estadía, principalmente cuando no existe un claro control de las autoridades públicas sobre los derechos de esa población. Sin embargo, en nuestra muestra el $80 \%$ reside en la comunidad con familiares o solos, lo cual da cuenta de lo naturalizado que se encuentra este abordaje por parte de todos los actores implicados (médicos, familiares, personas con demencia).

En las ciencias sociales del campo de la salud es ampliamente aceptada la incidencia directa que tiene en una persona con determinada enfermedad la forma en cómo significa y entiende la misma (p.e. Behuniak, 2011). En el caso de Uruguay, parecería que esta restricción química para controlar las conductas y sufrimiento de las personas es parte de una concepción que se basa en gestionar químicamente el funcionamiento de un órgano enfermo - en este caso el cerebro - como si el mismo fuera algo independiente de la persona. Esto responde a un esquema teórico que concibe a la enfermedad mental como un ente objetivo natural (Galende, 2008; Pérez, 2016; Stolkiner, 2013). En el caso de las demencias, esta concepción orienta fácilmente a que todos los problemas vinculares y familiares sean interpretados como un desajuste neuroanatómico y bioquímico, sin tener en cuenta lo señalado por autores clásicos sobre los efectos malignos del entorno y su incidencia en la neuropatología (Sabat, 2006). La visión lineal de que lo bioquímico produce efectos en el entorno, sin 
complejizar que esto es dinámico y que el entorno también produce efectos en lo bioquímico y en la patología, instala prácticas de gestión del órgano cerebral en la que se disuelve la persona. Se asiste así a la expresión de un dispositivo biopolítico altamente desubjetivante (Pérez, 2016).

Desde la perspectiva de la enfermedad como una entidad objetivo-natural, la misma se presenta como una verdad esencial, a-histórica y a-temporal, por fuera de las personas y sus modos de significar y vivir determinada condición de salud (Stolkiner, 2013). En una sociedad occidental en la que predomina una neurocultura (Higgs \& Gilleard, 2017), esta presentación como verdad ha llevado a una especie de vacío epistemológico en el tema, que es lo que habilita a que los supuestos subyacentes alrededor de la práctica de atención medicalizada sean presentados como certezas. A su vez, estas certezas, han llevado al SNIS a una especie de callejón sin salida para lograr prácticas acordes a los estándares de calidad.

Tanto los médicos entrevistados como las familiares están convencidos de que esta concepción de la enfermedad es el único universo probable. La producción de estas formaciones subjetivas remite a la compleja dinámica de prácticas discursivas y extradiscursivas sostenidas en lo macrosocial - en el imaginario social - que se reproducen en el plano microsocial - en el sujeto y sus vínculos -, en una continua retroalimentación. En la medida que es un circuito portador de emblemas identificatorios que cristalizan sentidos, produce también efectos precisos en su cuerpo y en la identidad. En el caso de las personas con demencia, estas prácticas las introducen en un proceso de desubjetivación, por el que cada vez son más pacientes enfermos y menos persona. Proceso nada nuevo si tenemos en cuenta lo planteado por Goffman, 1961/2001, sobre la carrera moral en que quedan capturadas las personas con enfermedad mental, y donde van aprendiendo a ser pacientes y ubicarse dentro del sistema desde el lugar de enfermo. Así, las prácticas instaladas forman 
parte de la creencia - que se convierte en profecía - sobre un destino inexorable, que se instala desde el primer tratamiento, desde el primer fármaco indicado, desde la primera acción de sujeción química.

Sin embargo, desde el campo de la salud mental comunitaria existe suficiente literatura crítica que permite considerar la salud y la enfermedad no como entidades absolutas, sino como procesos complejos (Galende, 2008; Stolkiner, 2013). Al respecto, resulta muy difícil sostener que una persona es totalmente sana o totalmente enferma mentalmente. Parecería más adecuado plantear que una persona tiene aspectos mentales más saludables y otros más patológicos que pueden coexistir y expresarse de diferente forma como respuesta a diferentes situaciones del diario vivir. Desde esta perspectiva teórica, el desarrollo de estos procesos no podrá entenderse por fuera de las prácticas socio-históricas que le dan sentido, principalmente las prácticas de atención y cuidado, que son las que fundamentan introducir los tratamientos psicosociales como primera línea de intervención. Sin duda otra sería la calidad y eficacia de la atención de las personas con demencia si esto se implementara así y los fármacos oficiaran como una segunda o tercera línea de apoyo. Ya finalizando este trabajo, es necesario señalar que sus resultados deben tomarse con cautela debido a algunas limitaciones del estudio. La primera de ellas es propia de la situación existente en Uruguay de dispersión de los datos sobre los diagnósticos y modalidades de atención a las personas con demencia. Debido a ello, se ha tenido que utilizar un criterio muy amplio de demencia, que en futuros trabajos se debería revisar. No es lo mismo lo que puede demandar en cuanto a estrategias de atención y cuidado una persona con demencia fronto-temporal, que otra persona con demencia vascular u otra con demencia tipo Alzheimer, etc. Por la misma razón, tampoco se pudo acceder a datos respecto a la etapa de avance de la demencia en que se encontraban las personas al momento de la entrevista. No obstante, hasta que no se 
implemente una política central de registro epidemiológico de esos datos, este estudio presenta una primera aproximación a la situación global. También constituye una limitación el tamaño de la muestra. Si bien el mismo es congruente con los tamaños muestrales que han utilizado otros estudios similares con entrevistas retrospectivas (p.e. Boise, et. al., 1999; Cattel et. al., 2000; Chrisp et. al., 2011; Speechly et. al., 2008), en el futuro trabajar con muestras mayores - y si fuera posible con estudios epidemiológicos - fortalecería mucho los resultados cuantitativos de asociación de variables y grupos.

Asimismo deben considerarse algunas fortalezas del presente trabajo. La primera de ellas es la de constituirse en el primer estudio de Uruguay y de América Latina que investiga este tema con el enfoque de trayectorias de atención y entrevistas retrospectivas. Esto permite definir una línea de base de la situación de atención a las personas con demencia para evaluar en el futuro la evolución de la misma. El diseño de doble triangulación (metodológica y de poblaciones) también fortalece la calidad de los resultados obtenidos, pues permite obtener datos numéricos (descriptivos e inferenciales) que abordan diferentes aspectos del problema, siendo los mismos profundizados cualitativamente para entenderlos e interpretarlos en las condiciones de producción de los hechos, desde los discursos de sus propios actores.

\section{Conclusiones}

El presente estudio permite sostener que la actual situación de atención a las personas con demencia en el sistema de salud de Uruguay, no cumple con los estándares de calidad definidos internacionalmente como buenas prácticas. Tal cómo se encuentran organizados actualmente los tratamientos, presentan un alto riesgo de producir efectos iatrogénicos. Para que el país pueda alcanzar satisfactoriamente las metas definidas por la OMS en el área de tratamientos y atención del plan de acción 
mundial sobre la respuesta de salud pública a la demencia, es necesario realizar al menos dos cambios centrales en las modalidades de atención: a) implementar tratamientos psicosociales de calidad, que estén disponibles como parte de las prestaciones del sistema de salud y del sistema de cuidados; b) generar estrategias de formación médica y de los equipos de salud, que permitan concientizar sobre los efectos del estigma en las demencias, a la vez que actualizarse en lo que respecta a las concepciones de salud mental comunitaria de estos trastornos, trascendiendo el modelo biomédico lineal, e incluir en los abordajes una perspectiva de Derechos Humanos que permita cuestionar el uso de los fármacos como sujeción química. Estos cambios permitirían modificar la actual forma de atención a las personas con demencias, abandonando las certezas inoperantes de la gestión química de los conflictos, para construir nuevas interrogantes clínicas sobre cada caso.

\section{Referencias}

Allegri, R., Arizaga, R., Bavec, C., Colli, L., Demey, I., Fernández, MC., ... Zuin, D. (2010). Guía de Práctica Clínica Enfermedad de Alzheimer. Buenos Aires: Sociedad Neurológica Argentina.

Alzheimer's Disease International. (2015). World Alzheimer Report 2015. The global impact of dementia: an analysis of prevalence, incidence, cost and trends. London: Alzheimer's Disease International.

Alzheimer's Disease International. (2013). La demencia en América: el coste y la prevalencia del Alzheimer y otros tipos de demencia. London: Alzheimer's $\begin{array}{lll}\text { Disease International. } & \text { Recuperado de }\end{array}$ https://www.alz.co.uk/sites/default/files/pdfs/dementia-in-the-americasSPANISH.pdf 
Alzheimer's Society. (2014). Dementia 2014: Opportunity for change. London: Alzheimer's Society.

Alzheimer's Society. (2013). The dementia guide. Living well after diagnosis. London: Alzheimer's Society.

Alzheimer's Society. (2011). Optimising treatment and care for people with behavioural and psychological symptoms of dementia. A best practice guide for health and social care professionals. London: Alzheimer's Society. Recuperado de http://www.alzheimers.org.uk/site/scripts/download_info.php?downloadID=609

Ballard, C.G., Waite, J. \& Birks, J. (2012). Atypical antipsychotics for aggression and psychosis in Alzheimer's disease (Review). The Cochrane Library, 1(CD003476). doi: 10.1002/14651858.CD003476.pub2

Banerjee, S., Hellier, J., Romeo, R., Dewey, M., Knapp, M., Ballard, C., ... \& Burns, A. (2013). Study of the use of antidepressants for depression in dementia: the HTA -SADD trial - a multicentre, randomised, double-blind, placebo-controlled trial of the clinical effectiveness and cost-effectiveness of sertraline and mirtazapine. Health Technology Assessment, 17(7). doi: 10.3310/hta17070

Bangash, A., Stubbs, R., Khan, F., Samnani, Sh., Aziz, H., \& Mitra, M. (2017). Association between antipsychotics and adverse outcomes in dementia. Progress in Neurology and Psychiatry, 21(4), 20-26. doi:10.1002/pnp.482

Behuniak, S. (2011). The living dead? The construction of people with Alzheimer's disease as zombies. Ageing and Society, 31(1), 70-92. doi:10.1017/S0144686X10000693

Biehl, J. (2008). Antropologia do devir: psicofármacos - abandono social - desejo. Revista de Antropologia, 51(2), 413-449. doi: 10.1590/S003477012008000200002 
Boise, L., Morgan, D., Kaye, J., \& Camicioli, R. (1999). Delays in the diagnosis of dementia: Perspective of family caregivers. American Journal of Alzheimer's Disease and Other Dementias, 14(1), 20-26. doi: 10.1097/WAD.0b013e3181a6bebc

Brasure, M., Jutkowitz, E., Fuchs, E., Nelson, VA., Kane, RA, Shippee, T., ... \& Kane, R. (2016). Nonpharmacologic Interventions for Agitation and Aggression in Dementia. Comparative Effectiveness Review (177). Recuperado de https://www.ncbi.nlm.nih.gov/pubmedhealth/PMH0086862/pdf/PubMedHealth PMH0086862.pdf

Brodaty, H. \& Arasaratnam, C. (2012). Meta-analysis of nonpharmacological interventions for neuropsychiatric symptoms of dementia. Am J Psychiatry, 169(9), 946-53. doi: 10.1176/appi.ajp.2012.11101529.

Burns, A., Rossor, M., Hecker, J., Gauthier, S., Petit, H., Möller, H., ... \& the International Donepezil Study Group. (1999). The Effects of Donepezil in Alzheimer's Disease - Results from a Multinational Trial. Dement Geriatr Cogn Disord., (10), 237-244. doi: 10.1159/000017126

Cabrera, E., Sutcliffe, C., Verbeek, H., Saks, K., Soto-Martin, M., Meyer, G.,... \& RightTimePlaceCare Consortium, T. (2015). Non-pharmacological interventions as a best practice strategy in people with dementia living in nursing homes. A systematic review. European Geriatric Medicine, 6(2), 134-150. doi: 10.1016/j.eurger.2014.06.003

Cammisuli, D.M., Danti, S., Bosinelli, F., \& Cipriani, G. (2016). Non-pharmacological interventions for people with Alzheimer's Disease: A critical review of the scientific literature from the last ten years. Eur Geriatr Med. 7(1), 57-64. doi: https://doi.org/10.1016/j.eurger.2016.01.002. 
Cattel, C., Gambassi, G., Sgadari, A., Zuccalà, G., Carbonin, P., \& Bernabei, R. (2000). Correlates of Delayed Referral for the Diagnosis of Dementia in an Outpatient Population. Journal of Gerontology Medical Sciences, 55(2), 98-102. doi: 10.1093/gerona/55.2.M98

Cheston, R. \& Ivanecka, A. (2017). Individual and group psychotherapy with people diagnosed with dementia: a systematic review of the literature. Int $J$ Geriatr Psychiatry, 32(1), 3-31. doi: 10.1002/gps.4529.

Chrisp, T., Thomas, B., Goddard, W. \& Owens, A. (2011). Dementia timeline: Journeys, delays and decisions on the pathway to an early diagnosis. Dementia, 10(4), 555-570. https://doi.org/10.1177/1471301211409375

Cooper, C., Mukadam, N., Katona, C., Lyketsos, C., Ames, D., Rabins, P., ... \& Livingston, G. (2012). Systematic review of the effectiveness of nonpharmacological interventions to improve quality of life of people with dementia. International Psychogeriatrics, 24(6), 856-870. doi: $10.1017 / \mathrm{S} 1041610211002614$

Cooper, C., Mukadam, N., Katona, C., Lyketsos, C., Blazer, D., Ames, D., ... \& Livingston, G. (2013). Systematic Review of the Effectiveness of Pharmacologic Interventions to Improve Quality of Life and Well-being in People With Dementia. The American Journal of Geriatric Psychiatry, 21(2), 173-183. doi: 10.1016/j.jagp.2012.10.018

Department of Health. (2009). Living well with dementia: A National Dementia Strategy. London: Department of Health. Recuperado de https://assets.publishing.service.gov.uk/government/uploads/system/uploads/att achment_data/file/168220/dh_094051.pdf

Drebing, Ch., Movitz, R., Lyon, P., Harden, T., McCarty, E. \& Herz, L. (2004). Documenting pathways to dementia care: Relative validity of questionnaire, 
interview, and medical record formats. American Journal of Alzheimer's Disease and Other Dementias, 19(3), 187-197. doi: 10.1177/153331750401900306

Galende, E., (2008). Psicofármacos y salud mental: la ilusión de no ser. Buenos Aires: Lugar.

García-Alberca, J.M. (2015). Las terapias de intervención cognitiva en el tratamiento de los trastornos de conducta en la enfermedad de Alzheimer. Evidencias sobre su eficacia y correlaciones neurobiológicas. Neurología, 30(1), 8-15. doi: 10.1016/j.nrl.2012.10.002

Gater, R., Almeida E. Sousa, D., Barrientos, G., Caraveo, J., Chandrashekar, C., Dhadphale, M., . . Sartorius, N. (1991). The pathways to psychiatric care: A cross-cultural study. Psychological Medicine, 21(3), 761-774. doi:10.1017/S003329170002239X

Goffman, E. (2001). Internados. Ensayos sobre la situación social de los enfermos mentales. Buenos Aires: Amorrortu. (Original publicado en 1961).

Higgs, P., \& Gilleard, Ch. (2017). Ageing, dementia and the social mind: past, present and future perspectives. Sociology of Health \& Illness, 39(2), 175-181. doi: 10.1111/1467-9566.12536.

Homma, A., Imai, Y., Tago, H., Asada, T., Shigeta, M., Iwamoto, T., ... \& Ohbayashi, T. (2008). Donepezil Treatment of Patients with Severe Alzheimer's Disease in a Japanese Population: Results from a 24-Week, Double-Blind, PlaceboControlled, Randomized Trial. Dement Geriatr Cogn Disord, 25(5),399-407. DOI: $10.1159 / 000122961$

Human Rights Watch. (2018). "They Want Docile". How Nursing Homes in the United States Overmedicate People with Dementia. [Nueva York]: Human Rights Watch 
Instituto Nacional de Estadística. (2018). Indicadores demográficos. Recuperado de http://www.ine.gub.uy/c/document_library/get_file?uuid=a70bf11d-a2a0-4f26aadb-fe4df9b5fc8b\&groupld=10181

Kirsch, I., Deacon, B., Huedo-Medina, T., Scoboria, A., Moore, T., \& Johnson, B. (2008). Initial severity and antidepressant benefits: a meta-analysis of data submitted to the Food and Drug Administration. PloS Med, 5(2), e45. doi:10.1371/journal.Pmed.0050045

Livingston, G., Kelly, L., Lewis-Holmes, E., Baio, G., Morris, S., Patel, N., ... \& Cooper, C. (2014). A systematic review of the clinical effectiveness and costeffectiveness of sensory, psychological and behavioural interventions for managing agitation in older adults with dementia. Health Technol Assess, 18(39),1-226. Doi: 10.3310/hta18390

Ministerio de Sanidad, Política Social e Igualdad de España. (2010). Guía de Práctica Clínica sobre la Atención Integral a las Personas con Enfermedad de Alzheimer y otras Demencias. Catalunya: Ministerio de Ciencia e Innovación.

National Institute for Health and Clinical Excellence. (2007). A NICE-SCIE Guideline on supporting people with dementia and their carers in health and social care. London: The British Psychological Society \& The Royal College of Psychiatrists Olazarán, J., Reisberg, B., Clare, L., Cruz, E., Peña, J., del Ser, T., ... Muñiz, R. (2010). Eficacia de las terapias no farmacológicas en la enfermedad de Alzheimer: una revisión sistemática. Dement Geriatr Cogn Disord, (30), 161178. doi: $10.1159 / 000321458$

O’Neil, M., Freeman, M., Christensen, V., Telerant, A., Addleman, A., \& Kansagara, D. (2011). Non-pharmacological Interventions for Behavioral Symptoms of Dementia: A Systematic Review of the Evidence. VA-ESP Project \#05-225. Recuperado de: 
https://www.ncbi.nlm.nih.gov/pubmedhealth/PMH0010206/pdf/PubMedHealth_ PMH0010206.pdf

Organización Mundial de la Salud. (2017). Plan de acción mundial sobre la respuesta de salud pública a la demencia, para el período 2017-2025. Recuperado de http://apps.who.int/gb/ebwha/pdf_files/WHA70/A70_28-sp.pdf?ua=1\&ua=1

Organización Mundial de la Salud. (2013). Demencia: Una prioridad de salud pública. Washington, DC: OPS. Recuperado de http://apps.who.int/iris/bitstream/10665/98377/1/9789275318256_spa.pdf

Pérez, R. (2016). Las dolencias de la mente. Prácticas de atención y cuidado de personas con demencia en Uruguay (Tesis de Doctorado inédita). Departamento de Salud Comunitaria, Universidad Nacional de Lanús, Remedios de Escalada, República Argentina.

Richter, T., Meyer, G., Möhler, R., \& Köpke, S. (2012). Psychosocial interventions for reducing antipsychotic medication in care home residents. Cochrane Database of Systematic Reviews, (12). Recuperado de https://www.cochranelibrary.com/cdsr/doi/10.1002/14651858.CD008634.pub2/fu II

Sabat, S. (2006). Mind, meaning and personhood in dementia: The effect of positioning. In: Hughes, J., Louw, S. and Sabat S, (editors) Dementia: Mind, meaning and the person (pp. 287-302). New York: Oxford University Press.

Sink, K., Holden, K., \& Yaffe, K. (2005). Pharmacological Treatment of Neuropsychiatric Symptoms of Dementia. A Review of the Evidence. JAMA, 293(5), 596-608. doi: 10.1001/jama.293.5.596

Sociedad Española de Geriatría y Gerontología. (2014). Documento de consenso sobre sujeciones mecánicas y farmacológicas. Madrid: SEGG. Recuperado de: 
https://www.segg.es/media/descargas/Documento_de_Consenso_sobre_Sujeci ones.pdf

Speechly, C., Bridges-Webb, Ch. \& Passmore, E. (2008). The pathway to dementia diagnosis. Medical Journal of Australia, 189(9), 487-489.

Stolkiner, A. (2013) Medicalización de la vida, sufrimiento subjetivo y prácticas en salud mental. Recuperado

de http://www.trabajosocial.unlp.edu.ar/uploads/docs/stolkiner_2013_medicalizacin _de_la_vida_sufrimiento_subjetiv_2014.pdf. Capítulo de libro en prensa

Zhao, M., Lv, X., Tuerxun, M., He, J., Luo, B, Chen, W., ... \& Wang, H. (2016). Delayed help seeking behavior in dementia care: preliminary findings from the Clinical Pathway for Alzheimer's Disease in China (CPAD) study. International Psychogeriatrics, 28(2), 211-219. doi: 10.1017/S1041610215000940

Formato de citación

Pérez, R. (2018). El tratamiento de las demencias en el sistema de salud de Uruguay. Psicología, Conocimiento y Sociedad, 8(2), 139-169. doi:

http://dx.doi.org/10.26864/PCS.v8.n2.8 